\title{
Event-Ready Bell Test Using Entangled Atoms Simultaneously Closing Detection and Locality Loopholes
}

\author{
Wenjamin Rosenfeld, ${ }^{1,2,{ }^{*}}$ Daniel Burchardt, ${ }^{1}$ Robert Garthoff, ${ }^{1}$ Kai Redeker, ${ }^{1}$ \\ Norbert Ortegel, ${ }^{1}$ Markus Rau, ${ }^{1}$ and Harald Weinfurter ${ }^{1,2}$ \\ ${ }^{1}$ Fakultät für Physik, Ludwig-Maximilians-Universität München, D-80799 München, Germany \\ ${ }^{2}$ Max-Planck Institut für Quantenoptik, D-85748 Garching, Germany
}

(Received 14 November 2016; revised manuscript received 13 February 2017; published 6 July 2017)

\begin{abstract}
An experimental test of Bell's inequality allows ruling out any local-realistic description of nature by measuring correlations between distant systems. While such tests are conceptually simple, there are strict requirements concerning the detection efficiency of the involved measurements, as well as the enforcement of spacelike separation between the measurement events. Only very recently could both loopholes be closed simultaneously. Here we present a statistically significant, event-ready Bell test based on combining heralded entanglement of atoms separated by $398 \mathrm{~m}$ with fast and efficient measurements of the atomic spin states closing essential loopholes. We obtain a violation with $S=2.221 \pm 0.033$ (compared to the maximal value of 2 achievable with models based on local hidden variables) which allows us to refute the hypothesis of local realism with a significance level $P<2.57 \times 10^{-9}$.
\end{abstract}

DOI: 10.1103/PhysRevLett.119.010402

Back in 1935 Einstein, Podolsky, and Rosen (EPR) pointed at inconsistencies in quantum mechanics, if one requires that a physical theory has to be realistic and local [1]. In such theories any signal, influence, or interaction propagates at most at the speed of light (locality), and one can assign properties to quantum systems before a measurement (realism). To achieve the latter, they left open the possibility to complement quantum mechanics with, nowadays called, local hidden variables (LHV). Starting from the EPR example on analyzing measurement results of two independent observers, John Bell showed that the prediction of QM for certain measurement scenarios differs from the prediction of all local, realistic theories [2]. With this he directly provided a prescription for how to evaluate the validity of the EPR claims and of any LHV theory in an experiment.

However, there are stringent requirements on an experimental test, as LHVs give a theory an amazing flexibility to account for observed results. In spite of the many experiments started soon after Bell's discovery (e.g., Refs. [3,4]), which (almost) all agreed well with QM, they all relied on assumptions on the observers or the observed systems, thus opening loopholes to the LHV theories under test (for reviews see, e.g., Refs. [5-7]).

One loophole, the locality loophole, concerns the independence of the observers, which only can be warranted if the whole measurement processes of the two observers are

Published by the American Physical Society under the terms of the Creative Commons Attribution 4.0 International license. Further distribution of this work must maintain attribution to the author(s) and the published article's title, journal citation, and DOI. spacelike separated. This was achieved by Weihs et al. [8], where the whole measurement, starting from the choice of a random number up to the appearance of the classical voltage signal of a single photon detection was outside the light cone of the other measurement. However, as detection of single photons was notoriously inefficient in those days, one had to assume fair sampling, i.e., that the registered photon pairs had been a representative sample of all pairs - thus leaving open the so-called detection loophole. This was closed for the first time in an experiment using trapped, entangled ions [9], which, however, were separated only by few micrometers-leaving the locality loophole open. Since then the goal was to close both in a single experiment, leading to key developments, such as the first observations of atom-photon entanglement $[10,11]$ and atom-atom entanglement over larger distances [12,13]. Recently, based on electron spins of separated nitrogenvacancy (NV) centers [14] the first experimental test of Bell's theorem without the locality and detection loophole was performed [15]. With the development of efficient photon pair sources [16] and highly efficient single photon detectors [17], two tests succeeded also with entangled photon pairs $[18,19]$.

Here we describe the evaluation of LHV theories using entangled neutral atoms closing both the locality and the detection loophole in a single experiment. Based on atomphoton entanglement, entanglement swapping [20] allowed us to prepare in a heralded manner entangled spin states of two atoms separated by a distance of $398 \mathrm{~m}$, well suited for an event-ready test. For an event-ready test no fair sampling assumption has to be made $[20,21]$. There a measurement result is reported every time the heralding signal confirming the successful distribution of entanglement to the observers 

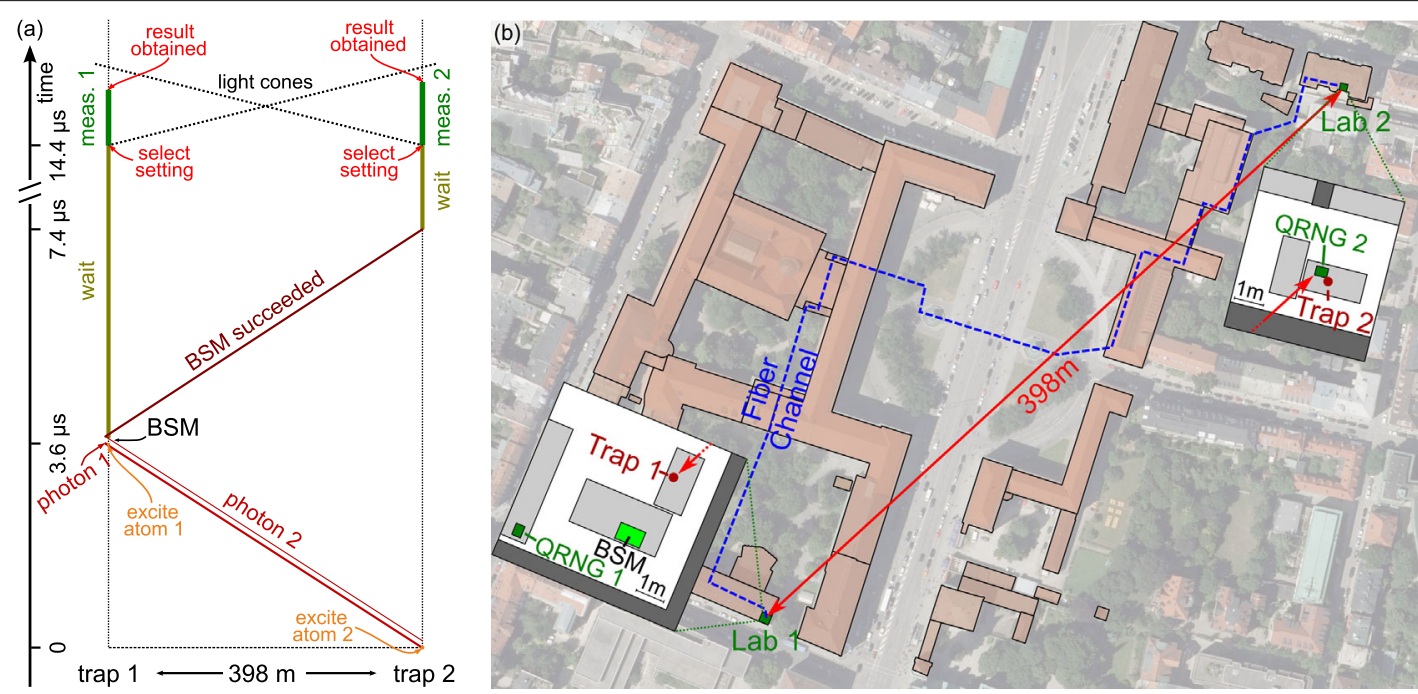

FIG. 1. (a) Space-time diagram of the experiment. The two observers (trap 1 and trap 2) are separated by $398 \mathrm{~m}$ with the BSM setup being located close to trap 1. Single photons and all communication signals are transmitted via optical fibers (lengths vary around $700 \mathrm{~m}$ ) laid in cable ducts connecting the two stations. Sending a photon from trap 2 to the BSM takes roughly $3.6 \mu$ s (photons from both traps arrive within a window of $120 \mathrm{~ns}$ represented by two lines for earliest and latest emission). Another $3.7 \mu$ s are needed for communicating the success of the BSM back to trap 2. The state measurements (including random choice of the measurement direction) are performed such that a result is obtained outside of the light cone of the other side. (b) Overview of the experimental location on the main campus of LMU. Trap 1 is located in the basement of the faculty of physics and trap 2 in the basement of the department of economics. Map data were provided by Ref. [31].

was obtained and thus no detection loophole is opened at all. Any inefficiencies or inaccuracies in the atomic state detection then only influence the degree of achievable correlations. The locality loophole is closed by employing fast and efficient measurements of the atomic spin states at a sufficient distance together with fast quantum random number generators (QRNG) for selection of the measurement basis. We employed state-dependent ionization for highly efficient atomic state analysis and with a total observation time of about a microsecond also the spacelike separation could be warranted. Well-defined hypothesis tests with samples of 10000 observations clearly indicate that LHV theories do not allow a correct description of nature.

We consider the simplest situation of an event-ready Bell test, where two separate observers are told-according to a heralding signal-to report the result of two-outcome measurements $A, B \in\{\uparrow, \downarrow\}$ performed on each side (an example are measurements on spin- $-\frac{1}{2}$ particles). For a test of local realism the two observers choose their measurement directions from two possibilities $a \in\left\{\alpha, \alpha^{\prime}\right\}$ and $b \in$ $\left\{\beta, \beta^{\prime}\right\}$ and afterwards compare their results. For this situation Clauser, Horne, Shimony, and Holt (CHSH) put Bell's inequality in an experimentally friendly form [22]:

$$
S=\left|\left\langle\sigma_{\alpha} \sigma_{\beta}\right\rangle+\left\langle\sigma_{\alpha} \sigma_{\beta^{\prime}}\right\rangle\right|+\left|\left\langle\sigma_{\alpha^{\prime}} \sigma_{\beta}\right\rangle-\left\langle\sigma_{\alpha^{\prime}} \sigma_{\beta^{\prime}}\right\rangle\right| \leq 2,
$$

with correlators $\left\langle\sigma_{a} \sigma_{b}\right\rangle=\left(1 / N_{a, b}\right)\left(N_{a, b}^{\uparrow \uparrow}+N_{a, b}^{\downarrow \downarrow}-N_{a, b}^{\uparrow \downarrow}-\right.$ $\left.N_{a, b}^{\downarrow \uparrow}\right)$. Here $N_{a, b}^{A, B}$ denote the number of events with the respective outcomes $A, B$ for measurement directions $a, b$ and $N_{a, b}$ is the total number of events of the respective measurement setting. Quantum mechanics predicts a violation of this inequality when measurements are performed on maximally entangled states $\left|\Psi^{ \pm}\right\rangle=$ $(1 / \sqrt{2})(|\uparrow\rangle|\downarrow\rangle \pm|\downarrow\rangle|\uparrow\rangle)$ with certain measurement settings, e.g., $\alpha=0^{\circ}, \alpha^{\prime}=90^{\circ}, \beta=-45^{\circ}, \beta^{\prime}=45^{\circ}$. Angles $\alpha, \beta$ are defined here in the spin space.

In our case the two observer stations are independently operated setups (trap 1 and trap 2) that are equipped with their own laser and control systems. Their separation of 398 m (Fig. 1) makes 1328 ns available to warrant spacelike separation of the measurements. On each side we store a single ${ }^{87} \mathrm{Rb}$ atom in an optical dipole trap. The employed internal spin states $\left(|\uparrow\rangle_{z}\right.$ and $\left.|\downarrow\rangle_{z}\right)$ are the Zeeman states $\left|m_{F}=+1\right\rangle$ and $\left|m_{F}=-1\right\rangle$ of the ground level $5^{2} S_{1 / 2}, F=1$ [Fig. 2(a)]. Entanglement of the atoms is generated by first entangling the spin of each atom with the polarization of a single emitted photon [11]. The photons are guided to an interferometric Bell state measurement (BSM) setup (Fig. 2), located close to trap 1. It consists of a fiber beam splitter (BS) followed by polarizing beam splitters (PBS) in each of the output ports, where detection of photons is performed by four avalanche photodiodes (APDs). This setup allows us to distinguish two maximally entangled photon states. Thereby a two-photon coincidence in particular detector combinations (see Sec. I. B of the Supplemental Material [23], which includes Refs. [24-30]) heralds the projection of the atoms onto one of the states $\left|\Psi^{ \pm}\right\rangle=(1 / \sqrt{2})\left(|\uparrow\rangle_{x}|\downarrow\rangle_{x} \pm|\downarrow\rangle_{x}|\uparrow\rangle_{x}\right)$ [13], where $|\uparrow\rangle_{x}=$ $(1 / \sqrt{2})\left(|\uparrow\rangle_{z}+|\downarrow\rangle_{z}\right)$ and $|\downarrow\rangle_{x}=(i / \sqrt{2})\left(|\uparrow\rangle_{z}-|\downarrow\rangle_{z}\right)$. 

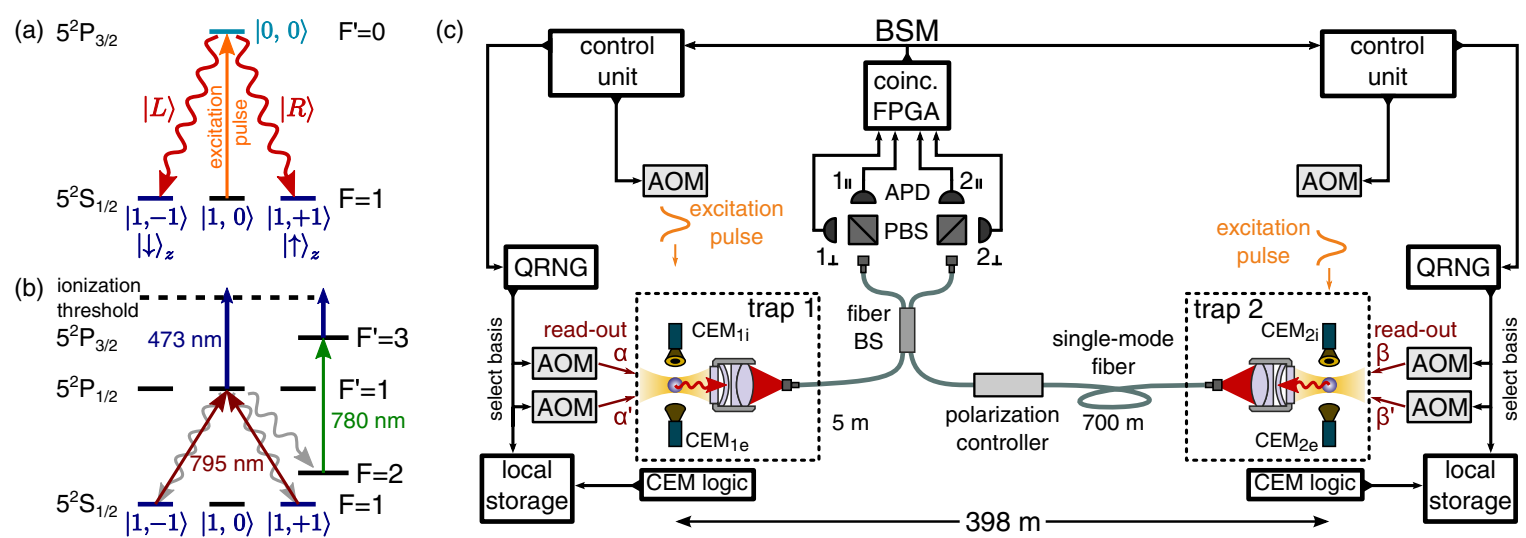

FIG. 2. (a) Scheme of the atomic levels involved in the entanglement between the spin state of the atom (subspace $5^{2} S_{1 / 2}, F=1$, $\left|m_{F}= \pm 1\right\rangle$ ) and polarization of the photon (left- and right-circular, $|L\rangle,|R\rangle$, respectively). Entanglement is generated in the spontaneous decay of the $5^{2} P_{3 / 2}, F^{\prime}=0$ state after optical excitation. (b) Scheme of the atomic state measurement. A selected superposition of the spin states is excited to the $5^{2} P_{1 / 2}, F^{\prime}=1$ level depending on the polarization of a $795 \mathrm{~nm}$ laser pulse and is ionized with a $473 \mathrm{~nm}$ laser. The atom can spontaneously decay to the $5^{2} S_{1 / 2}, F=1$ or $F=2$ levels during this procedure (gray wavy arrows). While decays into the $F=1$ level can reduce the fidelity of the measurement process, population in the $F=2$ level is excited with an additional $780 \mathrm{~nm}$ laser and ionized as well. (c) Schematic of the experimental setup. In each trap spin-polarization entanglement is generated between the atom and a single photon that is guided to the BSM via a single-mode fiber. Polarization stability in the $700 \mathrm{~m}$ fiber connecting trap 2 and the BSM is ensured by automatic compensation [32] performed every 5 min using reference light and a polarization controller. The photons are overlapped on a fiber beam splitter (BS), their coincident detection heralds entanglement of the atomic spins. Local measurements are performed on the atomic spins according to settings selected by quantum random number generators (QRNGs). Acousto-optic modulator (AOM), avalanche photo diode (APD), channel electron multiplier (CEM), field programmable gate array (FPGA), polarizing beam-splitter (PBS).

The experimental sequence (see Supplemental Material [23] Sec. I. B for further details) starts after two atoms are loaded into the traps. Photons emitted by the atoms are coupled into optical fibers. The efficiencies for detecting a single photon in the BSM arrangement after excitation in trap 1 or trap 2 are $\eta_{1}=1.65 \times 10^{-3}$ and $\eta_{2}=0.85 \times 10^{-3}$ [the latter also includes the transmission loss of photons $(\lambda=780 \mathrm{~nm})$ in the $700 \mathrm{~m}$ fiber of approximately $50 \%$ ]. This results in an overall probability to obtain a heralding signal in the BSM of $0.7 \times 10^{-6}$. If no signal is obtained the excitation sequence of the atoms is repeated. Including times necessary for transmission of signals as well as to prepare and to cool the atoms, the average rate of excitation attempts is $5.2 \times 10^{4} \mathrm{~s}^{-1}$. Depending on the loading rate of the traps this results in about $1-2$ heralding events per minute. The atom excitation procedures are synchronized to $<1 \mathrm{~ns}$ (Supplemental Material [23] Sec. I. A) such that the emitted photons entangled with the respective atoms have, at the BSM setup, a temporal overlap close to unity [13].

After a successful BSM signals are sent to both observers where they trigger the switching to atomic state measurement. An additional waiting time has to be introduced due to dephasing and rephasing of atomic states in strongly focused dipole traps. There, longitudinal field components lead to an inhomogeneous light polarization which results in a state- and position-dependent ac Stark shift. Because of the antisymmetry of the polarization distribution this accumulated phase is compensated for after one transverse oscillation [33]. To obtain simultaneous rephasing the radial trap frequencies are chosen for an oscillation period $\left(2 \pi / \omega_{r}\right)$ of 11.2 and $14.5 \mu \mathrm{s}$ for trap 1 and trap 2, respectively, by setting the trap depths. The measurement procedure starts with selecting the analysis direction according to the output of a fast quantum random number generator. As a further development of Ref. [34] these QRNGs have minimal bias (typically less than $10^{-5}$ ) without any postprocessing [35]. The random bit in trap 1 (trap 2) determining the direction $\alpha / \alpha^{\prime}\left(\beta / \beta^{\prime}\right)$ is provided on request and has no measurable correlation to bits generated earlier than 80 ns before; see Supplemental Material [23], Sec. II for details. In the sense of independence to previous information, we thus consider this moment before the request as the starting time of the measurement.

For the analysis of the atomic state a state-selective ionization is employed where the measurement direction $\gamma \in\left\{\alpha, \alpha^{\prime}, \beta, \beta^{\prime}\right\}$ is determined by the polarization of a readout laser at $795 \mathrm{~nm}$ exciting the atom to the $5^{2} P_{1 / 2}$, $F^{\prime}=1$ level from where it is ionized by an additional laser at $473 \mathrm{~nm}$ [Fig. 2(b)]. In particular, we ionize the state $|\uparrow\rangle_{\gamma}=\sin (\gamma / 2)|\uparrow\rangle_{x}-\cos (\gamma / 2)|\downarrow\rangle_{x}$ using linear polarization at an angle $\gamma / 2$ relative to the horizontal. The state $|\downarrow\rangle_{\gamma}=\cos (\gamma / 2)|\uparrow\rangle_{x}+\sin (\gamma / 2)|\downarrow\rangle_{x}$ remains unaffected. The resulting ${ }^{87} \mathrm{Rb}^{+}$ion and electron are accelerated by an electric field to two channel electron multipliers (CEMs) placed in $8 \mathrm{~mm}$ distance from the trapping region. The ionization fragments are detected with high efficiencies $\eta_{i}=0.90 \ldots 0.94$ (ions), $\eta_{e}=0.75 \ldots 0.90$ (electrons); the 
efficiencies are slightly different for the two labs and also vary between different measurement runs. We assign detection of at least one of the fragments to the atomic state $|\uparrow\rangle_{\gamma}$, providing a total detection efficiency of $\geq 0.98$ [36,37], while detection of no fragment is assigned to the state $|\downarrow\rangle_{\gamma}$. Note that in the event-ready scheme an imperfect detection efficiency does only affect the fidelity of the measurement process.

In order to perform a fast selection of the measurement direction we switch on one of two polarized readout laser beams with an acousto-optical modulator (AOM) (Fig. 2). The latency time from the output of the random bit of the QRNG until the readout pulse reaches the atom is 217 (204) ns. Optimizing the measurement fidelity we accept ions arriving at the detectors up to 570(725) ns after the beginning of the ionization process. The different times for the two traps result from different acceleration fields and, consequently, different times of flight of the ions. Together with the avalanche transition time within the CEMs and the latency of the processing electronics of 80(84) ns, the total time until the result appears as a digital pulse at the output is 947(1093) ns after the starting time of the measurement. We consider this signal being perfectly clonable and, thus, representing a definite classical entity with a value existing independent of observation. It is recorded together with the respective random bit (at trap 1 also with the result of the BSM) in a local storage unit.

We performed several measurement runs in the time period between November 2015 and June 2016. After a first clear violation with 300 events could be observed on Nov. 27 , 2015 (see Ref. [23], Sec. VI. A), the stability of the setup was improved allowing for long-term measurements. For testing the hypothesis that our experimental results can be described by a LHV theory, a well-defined experimental procedure was established to avoid expectation bias [38]. For that purpose all relevant details were fixed before the start of each run. These include the number of events to be collected, the analysis procedure, as well as scheduled maintenance to be performed; see the Supplemental Material [23], Sec. IV. We chose 5000 events for each prepared atomic state to achieve an appropriate level of significance, evaluation according to Eq. (1), and maintenance every $24 \mathrm{~h}$. We present two runs fulfilling these criteria in the following.

For the measurement run started on April 15, 2016 the obtained correlations are shown in Fig. 3. For the 5000 events for each of the two atom-atom states collected during 4 days, the resulting $S$ parameters of $2.240 \pm 0.047$ $\left(\left|\Psi^{-}\right\rangle\right)$and $2.204 \pm 0.047\left(\left|\Psi^{+}\right\rangle\right)$show a violation of the LHV limit by 5.1 and 4.3 standard deviations, respectively. By combining the events for the two atomic states we obtain $S=2.221 \pm 0.033$, corresponding to a violation by 6.7 standard deviations.

In order to determine the impact of these results for ruling out LHV theories we use the null hypothesis that the experiment is governed by LHV. Under this assumption
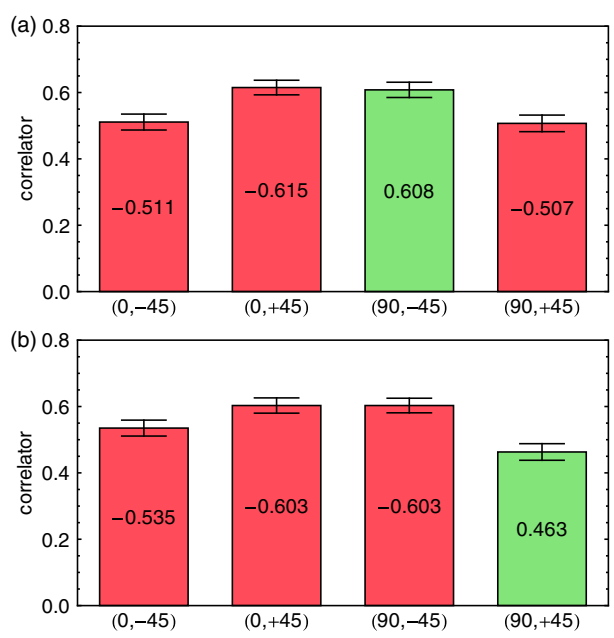

FIG. 3. Measured correlators $\left\langle\sigma_{a} \sigma_{b}\right\rangle$ for the run started on April 15,2016 for the atom-atom state $\left|\Psi^{-}\right\rangle$(a) and $\left|\Psi^{+}\right\rangle$(b). Displayed errors are equal to 1 standard deviation.

one can estimate the probability of obtaining a certain violation of Bell's inequality or a more extreme one, which is called the $P$ value. Within the hypothesis one can also allow for potential memory effects [39], where the history of the experiment may influence the probabilities of outcomes. We use two different models for calculating upper bounds for the $P$ value: the martingale approach [40] $\left(P_{m}\right)$ and the game formalism [41] $\left(P_{g}\right)$; for details see Ref. [23], Sec. III. For the combined data of the measurement above we obtain $P_{m}=2.57 \times 10^{-9}$ and $P_{g}=1.74 \times 10^{-10}$.

Explicit data for the above run, for the first violation in 2015 , as well as of further runs are documented in the Supplemental Material [23], Sec. VI. Especially, we want to point at the run started on June 14, 2016. The start of it was made public via the Twitter account [42] and simultaneously at a conference [43]. The results of each of the events, coming in at a rate of about $1 / \mathrm{min}$, were directly communicated to a central server [44], which made all the data available together with the momentary evaluation. In this public Bell test, due to the lower rate of trapping single atoms the $2 \times 5000$ events were collected during a time of 10 days, resulting in $S=2.134 \pm 0.048\left(\left|\Psi^{-}\right\rangle\right)$and $S=2.057 \pm 0.048\left(\left|\Psi^{+}\right\rangle\right)$. The violations of 2.8 and 1.2 standard deviations result in $P$ values for the combined data of $P_{m}=0.0267$ and $P_{g}=2.82 \times 10^{-3}$. It should be noted, that with the modest event rate the effect of the counting statistics on the momentary value of the $S$ parameter became clearly visible to a wide audience. The complete data are available for download from the server.

Finally, we consider a further frequently mentioned loophole-the free-will (or freedom of choice) loophole [45] targeting the independence of choice of the analysis directions from the hidden variables and vice versa [7]. Contrary to experiments with photon pairs $[18,19]$, eventready tests using entanglement swapping do not have a 
typical moment where the LHVs would have been defined [46]. If we assume that the LHVs are defined at the time of the BSM, in our experiment taking place $10.7 \mu$ s before the choice of the local analysis directions, they are clearly not influenced by the latter. Yet, contrary, the random settings are determined within the light cone of the BSM and independence has to be assumed here. This was accounted for in Refs. [15,18,19] where generation of the random numbers is considered being outside of the light cone of the entanglement generation (allowing to exclude influences within one trial of the experiment up to a few nanoseconds for the photon experiments $[18,19]$ or $690 \mathrm{~ns}$ for the experiment using NV centers [15]).

However, in the analysis of all experiments (including the present one) there is still the implicit assumption that the dependence of the random numbers generated for the $n$th observation event on processes or events of any kind in their backward light cone is strongly limited [47] (e.g., dependence on previous settings and outcomes of the experiment). Effectively, while one allows memory and by this dependence on the history for the LHV model determining the measurement outcomes, one does not allow memory for the (quantum) systems observed in the QRNGs to determine the settings. To avoid such assumptions-and the corresponding loopholes-and to warrant true independence of the random settings also in view of memory attributed to all quantum systems, one should produce random numbers outside the light cones of all other events of the Bell test. Spacelike separated extraterrestrial sources of randomness are required and have to be developed to ensure this [49].

In this Letter we described a highly reliable event-ready Bell test, showing in several attempts a clear violation of a Bell inequality. With violations of more than 6 standard deviations obtained in a run with 10000 events, the probability that this actual result could be described by local hidden variables is at most $P_{m}=2.57 \times 10^{-9}$. Taking all data accumulated during a time period of 7 months with over 55000 events (without any postselection) decreases this value to $P_{m}=1.02 \times 10^{-16}$. On the fundamental side, further reducing the number of assumptions on the independence of the randomness generation makes the development of methods for employing extraterrestrial sources highly desirable. From the point of view of applications, where the requirements for the random setting choice are different, our essentially loophole-free Bell test forms a promising platform for device-independent secure communication. The methods and results achieved here pave the way for new developments of quantum information and for future quantum repeater networks.

We gratefully acknowledge the help of all the people who contributed to the development of the experiment over the last 15 years, especially C. Kurtsiefer, M. Weber, J. Volz, F. Henkel, M. Krug, J. Hofmann, and we thank M. Zukowski for fruitful discussions. We acknowledge funding by the German Federal Ministry of Education and Research via the projects QuOReP and Q.com-Q, by the German-Israeli Foundation Project No. I-282-303.9-2013, and by the EU ERC Project QOLAPS (No. 291348).

*Corresponding author.

W.R@lmu.de

[1] A. Einstein, B. Podolsky, and N. Rosen, Can quantummechanical description of physical reality be considered complete?, Phys. Rev. 47, 777 (1935).

[2] J. S. Bell, On the Einstein-Podolsky-Rosen paradox, Physics 1, 195 (1964).

[3] S. J. Freedman and J. F. Clauser, Experimental Test of Local Hidden-Variable Theories, Phys. Rev. Lett. 28, 938 (1972).

[4] A. Aspect, J. Dalibard, and G. Roger, Experimental Test of Bell's Inequalities Using Time- Varying Analyzers, Phys. Rev. Lett. 49, 1804 (1982).

[5] J. F. Clauser and A. Shimony, Bell's theorem. Experimental tests and implications, Rep. Prog. Phys. 41, 1881 (1978).

[6] W. Tittel and G. Weihs, Photonic entanglement for fundamental tests and quantum communication, Quantum Inf. Comput. 1, 3 (2001).

[7] J.-A. Larsson, Loopholes in Bell inequality tests of local realism, J. Phys. A 47, 424003 (2014).

[8] G. Weihs, T. Jennewein, C. Simon, H. Weinfurter, and A. Zeilinger, Violation of Bell's Inequality under Strict Einstein Locality Conditions, Phys. Rev. Lett. 81, 5039 (1998).

[9] M. A. Rowe, D. Kielpinsky, V. Meyer, C. A. Sacket, W. M. Itano, C. Monroe, and D. J. Wineland, Experimental violation of a Bell's inequality with efficient detection, Nature (London) 409, 791 (2001).

[10] B. B. Blinov, D. L. Moehring, L.-M. Duan, and C. Monroe, Observation of entanglement between a single trapped atom and a single photon, Nature (London) 428, 153 (2004).

[11] J. Volz, M. Weber, D. Schlenk, W. Rosenfeld, J. Vrana, K. Saucke, C. Kurtsiefer, and H. Weinfurter, Observation of Entanglement of a Single Photon with a Trapped Atom, Phys. Rev. Lett. 96, 030404 (2006).

[12] D. L. Moehring, P. Maunz, S. Olmschenk, K. C. Younge, D. N. Matsukevich, L.-M. Duan, and C. Monroe, Entanglement of single-atom quantum bits at a distance, Nature (London) 449, 68 (2007); S. Ritter, C. Nölleke, A. Hahn, Reiserer, A. Neuzner, M. Uphoff, M. Mücke, E. Figueroa, J. Bochmann, and G. Rempe, An elementary quantum network of single atoms in optical cavities, Nature (London) 484, 195 (2012).

[13] J. Hofmann, M. Krug, N. Ortegel, L. Gérard, M. Weber, W. Rosenfeld, and H. Weinfurter, Heralded entanglement between widely separated atoms, Science 337, 72 (2012).

[14] F. Jelezko and J. Wrachtrup, Single defect centres in diamond: A review, Phys. Status Solidi A 203, 3207 (2006).

[15] B. Hensen et al., Loophole-free Bell inequality violation using electron spins separated by 1.3 kilometres, Nature (London) 526, 682 (2015).

[16] A. Fedrizzi, T. Herbst, A. Poppe, T. Jennewein, and A. Zeilinger, A wavelength-tunable fiber-coupled source of narrowband entangled photons, Opt. Express 15, 15377 
(2007); P. Trojek and H. Weinfurter, Collinear source of polarization-entangled photon pairs at nondegenerate wavelengths, Appl. Phys. Lett. 92, 211103 (2008).

[17] A. E. Lita, A. J. Miller, and S. W. Nam, Counting nearinfrared single-photons with $95 \%$ efficiency, Opt. Express 16, 3032 (2008).

[18] M. Giustina et al., Significant-Loophole-Free Test of Bell's Theorem with Entangled Photons, Phys. Rev. Lett. 115, 250401 (2015).

[19] L. K. Shalm et al., Strong Loophole-Free Test of Local Realism, Phys. Rev. Lett. 115, 250402 (2015).

[20] M. Zukowski, A. Zeilinger, M. A. Horne, and A. K. Ekert, "Event-Ready-Detectors" Bell Experiment via Entanglement Swapping, Phys. Rev. Lett. 71, 4287 (1993).

[21] J.S. Bell, Speakable and Unspeakable in Quantum Mechanics. (Cambridge University Press, Cambridge, England, 1988).

[22] J. F. Clauser, M. A. Horne, A. Shimony, and R. A. Holt, Proposed Experiment to Test Local Hidden-Variable Theories, Phys. Rev. Lett. 23, 880 (1969).

[23] See Supplemental Material at http://link.aps.org/ supplemental/10.1103/PhysRevLett.119.010402 for methods, data, and additional analysis.

[24] R. Glauber, Quantum Theory of Optical Coherence (WILEY-VCH, New York, 2007).

[25] R. Loudon, The Quantum Theory Of Light, 3rd ed. (Oxford Science Publications, New York, 1997).

[26] P. L'Ecuyer and R. Simard, TestU01: A C library for empirical testing of random number generators, ACM Trans. Math. Softw. 33, 22 (2007).

[27] Y. Zhang, S. Glancy, and E. Knill, Efficient quantification of experimental evidence against local realism, Phys. Rev. A 88, 052119 (2013).

[28] C. McDiarmid, in Surveys in Combinatorics (Cambridge University Press, Cambridge, England, 1989), Vol. 141, p. 148.

[29] A. Bednorz, Analysis of assumptions of recent tests of local realism, Phys. Rev. A 95, 042118 (2017).

[30] G. Adenier and A. Y. Khrennikov, Test of the no-signaling principle in the Hensen "loophole-free CHSH experiment", arXiv:1606.00784.

[31] Bayerisches Landesamt fuer Digitalisierung, Breitband und Vermessung

[32] W. Rosenfeld, F. Hocke, F. Henkel, M. Krug, J. Volz, M. Weber, and H. Weinfurter, Towards Long-Distance AtomPhoton Entanglement, Phys. Rev. Lett. 101, 260403 (2008).

[33] W. Rosenfeld, Ph. D. thesis, Ludwig-MaximiliansUniversity Munich, 2008, http://nbn-resolving.de/urn:nbn: de:bvb:19-100300; J. D. Thompson, T. G. Tiecke, A. S. Zibrov, V. Vuletic, and M. D. Lukin, Coherence and Raman Sideband Cooling of a Single Atom in an Optical Tweezer, Phys. Rev. Lett. 110, 133001 (2013); D. Burchardt et al. (to be published).
[34] M. Fürst, H. Weier, S. Nauerth, D. G. Marangon, C. Kurtsiefer, and H. Weinfurter, High speed optical quantum random number generation, Opt. Express 18, 13029 (2010).

[35] C. Abellan, W. Amaya, D. Mitrani, V. Pruneri, and M. W. Mitchell, Generation of Fresh and Pure Random Numbers for Loophole-Free Bell Tests, Phys. Rev. Lett. 115, 250403 (2015).

[36] F. Henkel, M. Krug, J. Hofmann, W. Rosenfeld, M. Weber, and H. Weinfurter, Highly Efficient State-Selective Submicrosecond Photoionization Detection of Single Atoms, Phys. Rev. Lett. 105, 253001 (2010).

[37] N. Ortegel, Ph. D. thesis, Ludwig-Maximilians-University Munich, 2016, http://nbn-resolving.de/urn:nbn:de:bvb: 19-199728.

[38] M. Jeng, A selected history of expectation bias in physics, Am. J. Phys. 74, 578 (2006).

[39] J. Barrett, D. Collins, L. Hardy, A. Kent, and S. Popescu, Quantum nonlocality, Bell inequalities, and the memory loophole, Phys. Rev. A 66, 042111 (2002).

[40] R. D. Gill, Time, finite statistics, and Bell's fifth position, arXiv:quant-ph/0301059v2.

[41] N. Brunner, D. Cavalcanti, S. Pironio, V. Scarani, and S. Wehner, Bell nonlocality, Rev. Mod. Phys. 86, 419 (2014); D. Elkouss and S. Wehner, (Nearly) optimal P values for all Bell inequalities, npj Quantum Inf. 2, 16026 (2016).

[42]@munichbellexp, https://twitter.com/munichbellexp

[43] Quantum and Beyond, International Conference Devoted to Quantum Theory and Experiment, Linnaeus University, Växjö, Sweden, June 13-16, 2016.

[44] http://bellexp.quantum.physik.uni-muenchen.de

[45] J. S. Bell, J. F. Clauser, M. A. Horne, and A. Shimony, An exchange on local beables, Dialectica 39, 85 (1985).

[46] C. Branciard, N. Gisin, and S. Pironio, Characterizing the Nonlocal Correlations Created via Entanglement Swapping, Phys. Rev. Lett. 104, 170401 (2010).

[47] There are well-developed descriptions accounting for a possible dependence of the random settings on the history of the experiment $[41,48]$ [see the Supplemental Material [23] Eq. (10)]. Yet, this dependence is effectively set to a very small value, only depending on technical noise evaluated within a physical model ([35] and Supplemental Material [23], Sec. II), and thus one effectively assumes independence of the generation process from the history.

[48] J. Kofler, M. Giustina, J.-A. Larsson, and M. W. Mitchell, Requirements for a loophole-free photonic Bell test using imperfect setting generators, Phys. Rev. A 93, 032115 (2016).

[49] J. Gallicchio et al., Testing Bell's Inequality with Cosmic Photons: Closing the Setting-Independence Loophole, Phys. Rev. Lett. 112, 110405 (2014); J. Handsteiner et al., Cosmic Bell Test: Measurement Settings from Milky Way Stars, ibid. 118, 060401 (2017). 